Sousa H.S., Branco, J. M., Lourenço, P. B. (2015, just accepted) A holistic methodology for probabilistic safety assessment of timber elements combining onsite and laboratory data. International Journal of Architectural Heritage. (http://dx.doi.org/10.1080/15583058.2015.1007177)

\title{
A holistic methodology for probabilistic safety assessment of timber elements combining onsite and laboratory data
}

Hélder S. Sousa ${ }^{\mathrm{a}, *}$ : Postdoctoral researcher

Jorge M. Branco ${ }^{\text {a }}$ : Assistant Professor

Paulo B. Lourenço ${ }^{\mathrm{a}}$ : Full Professor

${ }^{a}$ ISISE, Department of Civil Engineering, University of Minho, Portugal

* corresponding author: ISISE, University of Minho

Department of Civil Engineering, Azurém,

4800-058 Guimarães, Portugal

e-mail: sousa.hms@gmail.com

Tel: +351 253510200; Fax: +351253510217

\begin{abstract}
The assessment of existing timber structures comprises several fields of knowledge, each one providing its own piece of information aiming at an effective answer. Even if general guidelines are available, there is still a need for a holistic approach that is able to combine information from different sources and infer upon that information allowing also, in that process, for an updating scheme aiming at a more substantiated decision process. In that scope, this work proposes a methodology for a holistic assessment of timber elements. The methodology combines information gathered in different scales and follows a probabilistic framework that allows for the structural assessment of existing timber elements with possibility of inference and updating of the mechanical properties of the elements, through Bayesian methods. The described methodology is based in four main steps: (i) scale of information; (ii) local and global data; (iii) probability assignment; and (iv) long term structural analysis. After the description of the methodology, its limitations and possible future modifications are discussed.
\end{abstract}

KEYWORDS: Timber elements, assessment, methodology, reliability, Bayesian methods 


\section{INTRODUCTION}

The large material variability of timber can explain why the design of timber structures has often been based on experience and subjective engineering judgment, having led, in many cases, to conservative solutions. At the present state of knowledge, rational design rules based on detailed material description and validated by comparison with many empirical results are made available to a timber construction designer. However, the safety assessment of existing structures and the characterization of traditional wooden building techniques remain true challenges.

When dealing with existing timber structures, the structural safety assessment and evaluation of each component, either the element itself or its connections, and the system reliability must be determined according to the present and onsite conditions of the structure. On the other hand, maintenance plans, or when necessary repair or strengthening actions, must be considered within the framework of the lifecycle evaluation of the structure based on its predicted durability and vulnerability to exterior actions.

The assessment of existing timber structures comprises several fields of knowledge, each one providing its own piece of information, aiming at a better and more complete answer. Largescale timber specimens should be experimentally tested to determine the influence of each of those sources of information. However, it is often not possible to experimentally evaluate fullscale structures or elements due to onsite conditions, social or cultural restrictions or, even economic impediments. As a consequence, it is of interest to predict the behaviour of large-scale timber elements by consideration of the mechanical properties of small specimens complemented with information obtained at the global scale of the element. Therefore, inspection and evaluation of the onsite structural properties, many times using visual grading and non-destructive testing, represent an important step in the assessment of existing timber structures and have a significant role in their diagnosis, analysis and conservation. To that aim, the conception and implementation of methodologies for safety assessment of existing timber elements are needed, in particular through the analysis of small scale specimens aided by the information of visual grading and non-destructive testing. Although several methodologies provide definitions and guidelines for specific problems, there is still a need for a holistic approach able to combine information from different sources and infer upon that information, also allowing for an updating process and a sound decision process. Thus, this work proposes a methodology based on well-established premises, providing guidelines for a holistic assessment of timber elements. 


\section{PRESENT METHODOLOGY FOR ASSESSMENT}

The assessment of timber structures is often based on the experience of engineering judgement which provides it with a non-quantifiable subjective nature. To minimize that subjectivity, the conditions and state of conservation of the structure can be tested and evaluated through different methods.

In the case of heritage timber structures, a greater expense, both in the survey/diagnosis/assessment of the structure and in the repair methods that are employed, might be justified due to their historical significance and cultural value. Within that scope, the best possible assessment of its current structural characteristics should be considered, which may require the use of more precise and sophisticated methods than the commonly used, with associated costs that are unbearable for common structures. The framework for assessment of heritage timber structures was detailed in Cruz et al. (2013) as summarized in Figure 1. The method is mainly divided in three parts: (i) preliminary assessment; (ii) structural analysis and detailed assessment, and; (iii) assessment results and future actions.

The assessment and intervention on common timber structures is often based on visual inspection of the structure and consequent elaboration of damage maps or datasheets that indicate the segments or regions of the structure that are of higher structural concern and in need of intervention (Macchioni et al., 2012; Alessandri et al., 2012; Lourenço et al., 2013).

When further information is needed to better understand the actual conditions of the structure, non-destructive tests or even the removal and mechanical testing of small size specimens are possible. Nevertheless, these procedures only determine the condition of the structure at a given time period and often the whole assessment must be repeated to consider new information. In this case, the use of probabilistic methods allows for a continuous structural assessment able to incorporate the uncertainty of the assessment instruments, the subjectivity of the analysis and also to provide a consistent tool for updating the analysis with new information (Cointe et al., 2007; Zona et al., 2011; Sousa et al., 2013b). 


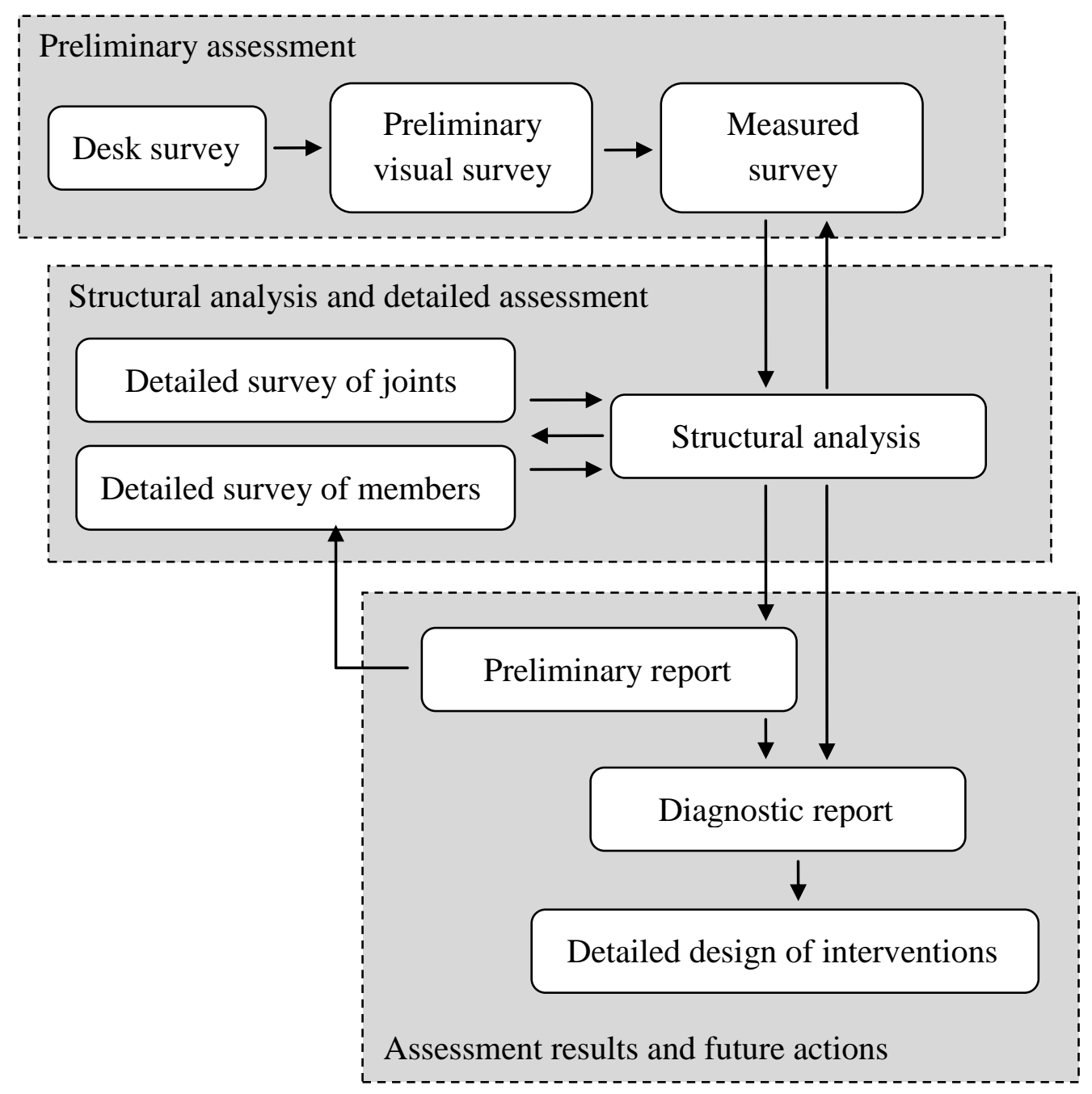

Figure 1: Steps required for the assessment and planning of interventions in heritage timber structures (adapted from Cruz et al., 2013).

\section{ASSESSMENT INSTRUMENTS}

The methodology proposed in this document is based on the combination of data taken from different scales, both at material and structural element levels. To that aim, the data is obtained through results of visual inspection and non-destructive tests complemented with mechanical testing of small size specimens.

Visual inspection is a global testing method used to identify the overall condition of the structure and wood members, and it is considered the most important step in the assessment process (Kasal, 2010). Several authors even mention that visual inspection is required to determine the original timber characteristics and the damage history during its service life (Ross et al., 1998; Tampone et al., 2002). Although it may be preceded by a preliminary desk survey, when doing, for example the historical survey of heritage structures, visual inspection 
is the first step to a full diagnostic of an existing structure and often a crucial factor that determines the plan of interventions (Cruz et al., 2013).

During visual inspection, the natural defects and deterioration are detected, characterized and inventoried. Natural defects include features such as knots, slope of grain, deformation, wane and seasoning checks, while deterioration includes damage from insect infestation or fungal decay (biologic attack). Besides the presence of defects in elements, it is crucial to survey the moisture content and/or water infiltrations, as those may contribute to the presence of biological activity. Special attention must be given to the connections between elements and its condition. The structural safety is highly dependent on the performance of the connections, however few standards address the visual inspection of connections with due concern.

After visual inspection, timber grading is made based on the size, number and location of defects related to the member's size and structural use. This grading is often controlled by a critical segment of the member and accounts for its residual cross section (section without the portion decayed by biotic agents). An example of this procedure is found in the Italian standard UNI 11119 (UNI, 2004). This standard establishes objectives, procedures and requirements for the diagnosis of the state of conservation and estimates the mechanical properties (resistance and stiffness) for structural wood elements present in cultural heritage buildings.

Several other testing methods were made available during the last decades, as to answer different questions regarding the characterization of timber. Depending on its nature, application and damage that they produce to the inspected elements, these tests are defined into different categories. One of the most important division is that which defines the amount of damage made to the material while performing the test, resulting into three categories (Kasal and Anthony, 2004). When a test does not produce damage it is denominated as nondestructive testing (NDT), or semi-destructive (SDT) when only minor damage is made. On the other side, destructive testing (DT) takes place when the material or element is damaged in such way that it cannot be recovered.

Although NDT has the advantage of maintaining the full integrity of the element, it does not provide a high level of information and a large amount of measurements are necessary to provide a preliminary material characterization. Only DT allows for a complete and reliable material characterization, even if it is usually inadequate in the assessment of historic structures. As in DT, the advantage of SDT is that the mechanical property under study is measured directly and is not obtained indirectly, whereas NDT relies on the relationship 
(often correlation) between the measured data and a mechanical parameter (either strength or stiffness).

Although, in an overall sense, all of these tests and procedures are intended to give a better understanding of the material characterization, they often individually provide only insight of a specific parameter or property. For instance, many NDTs require information provided by SDTs in order to correlate the data with the mechanical properties of timber (e.g. ultrasonic methods depend on the knowledge of density and moisture contents, obtained by SDT for a reliable estimation of timber's stiffness). Therefore, the timber assessment should comprise a thorough testing procedure, combining different tests and regarding a variety of parameters (e.g. dimensional stability, mechanical strength, durability or moisture content), so that timber can be correctly graded according to accepted professional guidelines or national regulations. With concern to existing structures, several methods have been established to evaluate timber onsite, and the choice of a specific method depends on the particular information that is required. Further information about individual test methods are summarized in the RILEM TC 215 state-of-the-art report (Kasal and Tannert, 2010). Detailed information on the onsite application of NDTs was provided in Riggio et al. (2014) and Dackerman et al. (2014), while onsite application of SDTs was dealt with in Tannert et al. (2014). These works cover important issues related to the onsite application of main testing methods, such as basics, equipment, field procedures, sampling, result interpretation and limitations.

\section{PROPOSED HOLISTIC METHODOLOGY}

\subsection{Methodology and application}

The combination of results from different tests and visual inspection can be used to determine the present condition of an existing timber element and to predict its structural performance. In Calderoni et al. (2006), the global influence of relevant defect patterns on the behaviour of chestnut floor beams was evaluated by comparing their overall behaviour with the actual material response obtained by compression, bending and shear tests on defect-free small specimens. Several other works have proposed this combination of information aiming at the evaluation of individual and global mechanical properties (Branco et al. 2010; Faggiano et al. 2011; Machado, 2013), and a probabilistic approach is often used (Fink and Köhler, 2011; Sousa, 2013; Fink and Köhler, 2014a), since these methods are prone to incorporate the uncertainty of the initial data and to update it with new information. However, a consistent 
and global framework allowing for the assessment, updating and intervention planning is yet to be established for the probabilistic evaluation of existing timber structures.

In this scenario, a holistic methodology for the assessment of timber elements is proposed by combining onsite and laboratory data with the determination of probabilistic parameters that are suitable for reliability analysis and updating through different Bayesian methods. The methodology is portrayed in Figure 2. The method is based on the determination of the material properties of clear wood specimens, and subsequently combined with information obtained through the analysis of defects by visual inspection and NDT, allowing to assess the element at a structural scale. This concept, although widely used in timber engineering (Fink and Köhler, 2011; Machado and Palma, 2011; Fink and Köhler, 2014a), is here used within a framework that allows the structural assessment of existing timber elements with possibility of inference and updating of the elements' mechanical properties.

The methodology is divided into four main steps. In the first two steps, the information is divided according to the scale of the element and the source of data. With these initial steps it is possible to implement the information that commonly is available to an engineer, and to divide it considering the material scale (clear wood) or the structural scale (element). Afterwards, the information is used to establish the reference properties, both local and global, in a probabilistic framework that allows for the prediction and inference of the mechanical properties. Finally, the results are used for the structural reliability assessment of the existing structure allowing for the definition of the intervention (if required) and the inclusion of monitoring and maintenance plans through the updating process. Next, a detailed description of each step is provided and explained through exemplifying diagrams. The results evidenced within the examples of each phase were based on the experimental campaigns and researches of Lourenço et al. (2013), Sousa (2013) and Sousa et al. (in-press). 


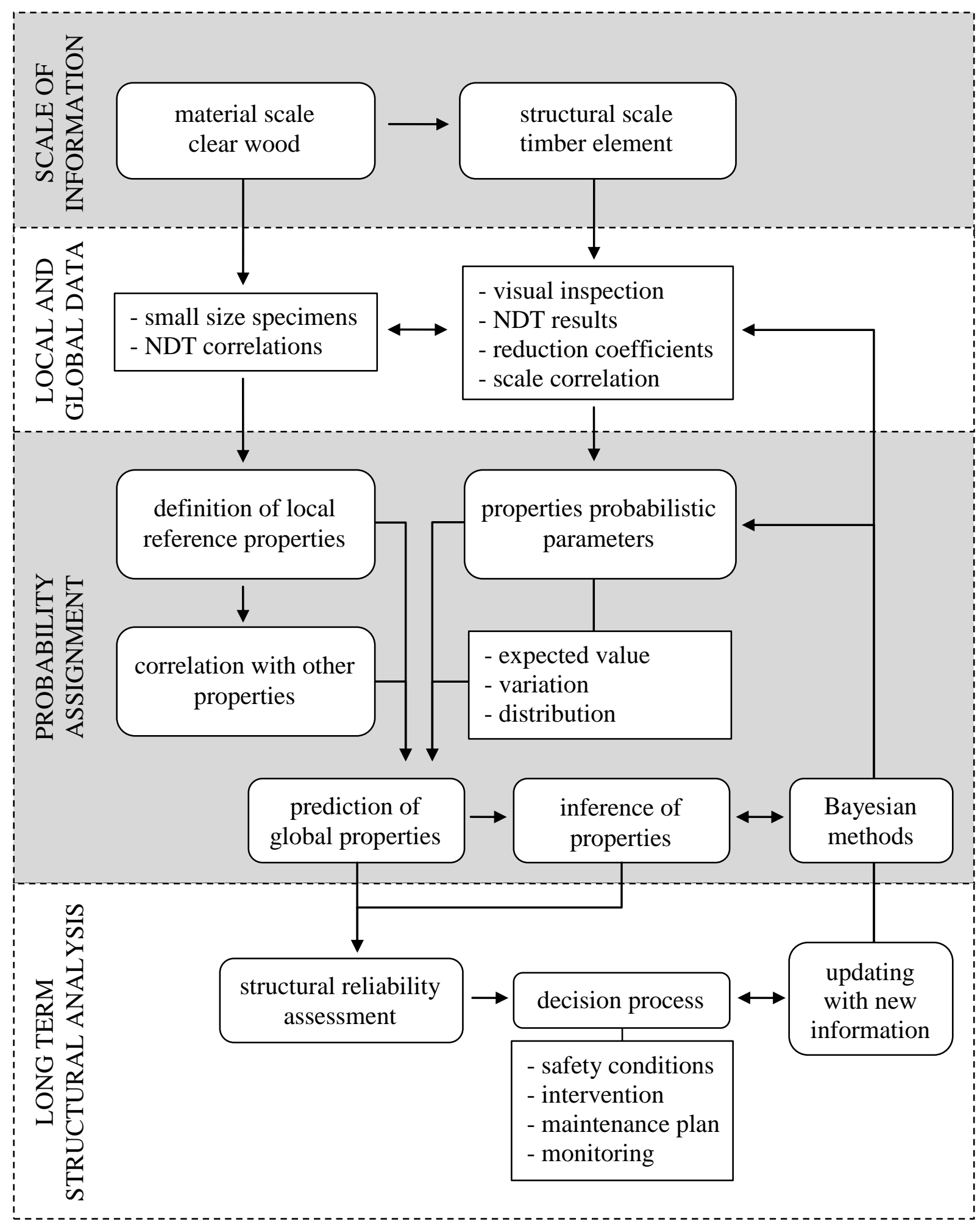

Figure 2: Holistic methodology for probabilistic safety assessment, inference and updating of timber elements combining information from different scales. 


\subsection{Scale of information}

Within this methodology, it is important to determine the scale to each source of information refers to. The variability in timber's mechanical properties may be divided according to different scales, allowing for a better definition of local or global properties affected by the amount of defects or condition. On this methodology, the scale of information does not only comprise the variation of size within elements in terms of geometric dimension (width, height, length and volume) or shape (round or rectangular cross section), but also it considers the state of conservation when distinguishing between apparent or residual cross section. Another premise of this step is that the information retrieved on smaller size scales is fundamental to the mechanical properties of larger size scales and, therefore, for the same element there is a dependency between results of different scales. In Figure 3 is showed an example of a hierarchic representation of different scales. In this case three scales are selected, considering from the state of conservation of onsite elements to the cross section scale. However, size and scale effects should be determined to correlate information between scales.

structural scale

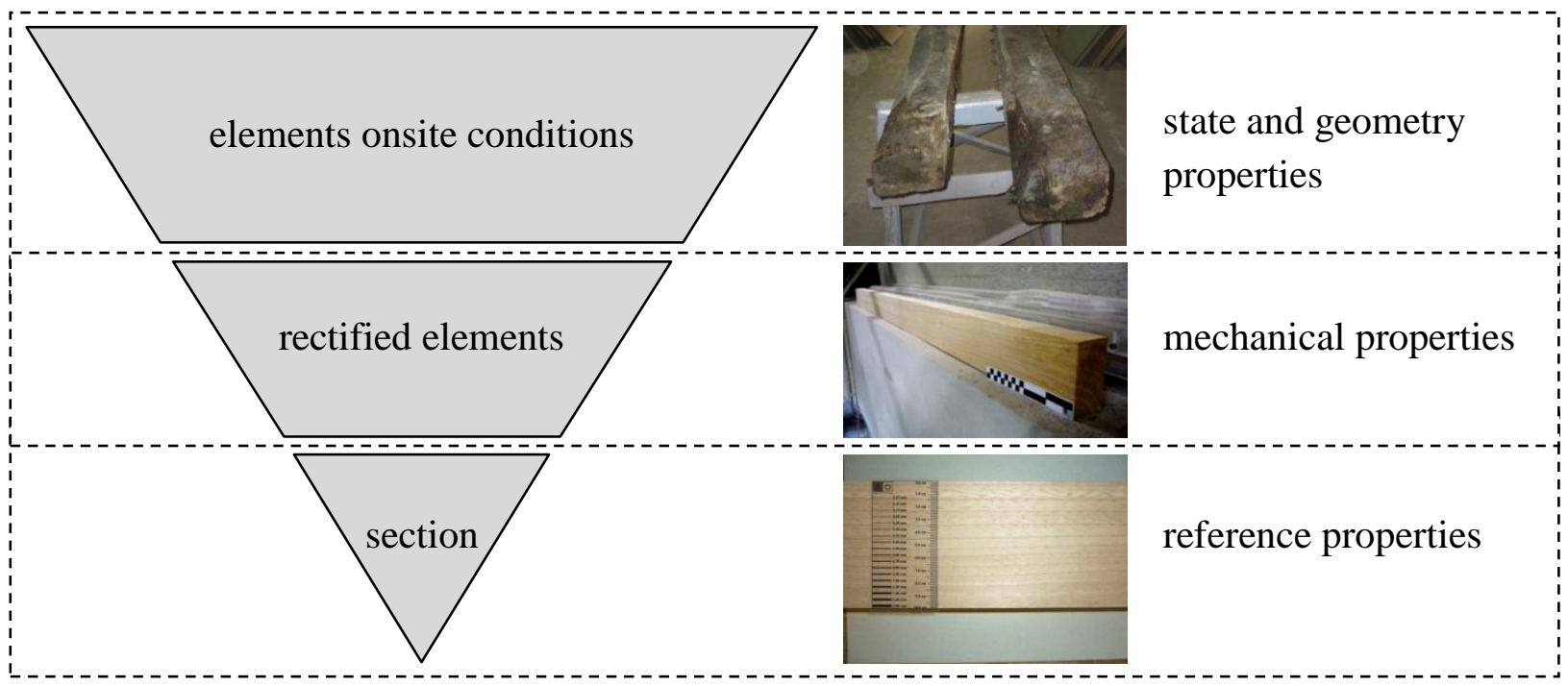

material scale

Figure 3: Hierarchical definition of information by scale.

Size, length and the distance between defects, are parameters that constrain the mechanical behaviour of the affected cross sections and nearby sections of a structural element. In this case, the properties of wood located between defects (clear wood) are expected to correspond to higher strength and stiffness, and to present less variability, when compared to sections of 
timber influenced by defects. Thus, it is not prudent to consider only the results of mechanical tests of small samples without defects, since its presence largely influences the element's structural behaviour.

At the element level, several attempts have been made to hierarchically model the stiffness and strength of timber members, by considering the presence of weak sections separated by segments of clear wood (Riberholt and Madsen, 1979; Isaksson, 1999; Fink and Köhler, 2011; Machado and Palma, 2011). Even for the same scale, the dimensions of a specific timber element affect the strength, since there is higher probability of having a weaker section for an element with larger length or with larger cross section. Size effect is already being considered in design regulations such as in the EN 1995-1-1 (CEN, 2004).

\subsection{Local and global data}

One of the principles of this proposed methodology is the combination of local data obtained at the material scale with global data obtained at the element scale, in order to evaluate the elements' performance at a structural level. To that aim it is essential to obtain proper information from both scales.

In the material scale, small size clear wood specimens may be taken from the structure as to assess the mechanical properties of the element when not influenced by defects or level of conservation, through NDT and DT. The results obtained by the analysis of these small clear wood specimens may, subsequently, be correlated with the results of NDT in order to indirectly obtain indication about other mechanical properties.

The extraction of small specimens is an SDT which accuracy depends on the adoption of appropriate sampling criteria. Small specimens are extracted from the structural member, such that its dimensions are smaller than wood's natural defects or significantly smaller than the member itself, ensuring that the strength of the member is not affected. In this case, regarding the variability within member, the number of measurements will greatly affect the reliability of the prediction of the desired parameter due to the different variability values from one size scale to another. Specimens should be taken from segments of the timber element without defects (clear wood specimens) but also from critical sections of the element, as to be representative of the sample (sections with higher loading conditions or with deterioration). Due to its small dimensions of the specimens, the sampling criteria must attend to the number of growth rings and to the proportionality between early and latewood. Recommendations 
regarding those parameters may be found in ASTM D143 (ASTM, 2000) regarding different methods for testing small clear specimens of timber.

The number of specimens and the location where they should be extracted depends on the mechanical property that is being analyzed and on the accuracy of the testing method itself. In a probabilistic assessment, the number of tests should be considered accordingly to the importance of the analysis and its objective. For instance, a minimum sample size of 40 specimens is recommended on ISO 13910 (ISO, 2005) for the determination of characteristic values. In other cases, as proposed in ISO 3129 (2012) the minimum number of specimens for extraction may be considered directly related to the coefficient of variation of the property in analysis. However, often a large number of samples is required in order to obtain a stable sample coefficient of variation.

In the case of existing timber elements, always a compromise between the desired reliability for the information and the needed amount of specimens should be considered as to avoid unnecessary extractions of timber specimens. Detailed information about different SDTs and its conditions of use, including sampling criteria, may be found in Tannert et al. (2014).

Currently, the extraction of small size specimens is a well-established procedure and several authors presented correlations of NDT with mechanical properties for small clear wood specimens (Lourenço et al. 2007; Feio et al. 2007; Calderoni et al., 2010). The information obtained through this approach often presents an upper limit of the actual mechanical properties of the element, and it is necessary to reduce these values attending to the defects and level of conservation of the element. To that aim, global testing such as visual inspection and other NDT results may be considered. In this case, visual inspection should comprise the analysis of the entire element, taking into account its state of conservation, loading conditions and existence of critical sections. Moreover, the inspection should also be sufficiently detailed as to distinguish the variation of the defects along the element's dimensions, thus defining and distinguishing between sections of clear wood and sections with defects of different sizes and importance. It is recommended that different visual grades are given to each section of the element, according to the defects (considering as example the knot size, misalignment of fibers and crack width), in order to evidence and estimate the possible variation of mechanical properties along the element. According to this visual grading, different reduction coefficients may be considered, which will downgrade the mechanical properties obtained for small clear wood specimens in proportion with the level of defectiveness. 
Another important aspect of this step, for the framework of the methodology, is that the data obtained is not only assessed within the same scale but it is also correlated to the data of different scales. For that, correlation between the different tests (sampling of clear wood specimens, visual inspection, NDT and others) should be considered between the different scales of the same element, as to provide a prior database for evaluation of elements to which only information on one of the scales was attained.

Figure 4 presents an example of this phase, considering the local information obtained through the correlation of mechanical tests in clear wood specimens and NDT (e.g. ultrasounds). In the work of Sousa (2013) different NDT were correlated with mechanical properties of chestnut timber elements at different scales. Results of ultrasound, pin penetration and drilling resistance tests were correlated with bending, compression and tension modulus of elasticity. The results of NDT were better correlated with bending stiffness achieving coefficients of determination $\left(r^{2}\right)$ above 0.80 , which represents a high correlation according to the proposed in the probabilistic model code for timber (JCSS, 2006). Results of coefficients of determination between NDTs and modulus of elasticity for old timber elements were compiled in Cavalli and Togni (2013). Also in that work, a testing approach similar to the considered in this example was considered. In that case, 13 old timber members of Silver Fir were visually graded and tested with different NDTs for the density and modulus of elasticity estimation. The tests comprised of flexural and longitudinal vibrational tests, stress wave transmission time and pin penetration depth. The timber elements were also tested in a f four-point bending tests to determinate the local and global modulus of elasticity. In that experimental campaign it was possible to divide the timber elements in three grades, through visual inspection, on the basis of the presence and extension of defects. Also the example presented in Figure 4 considered visual inspection as a global test and was used to differentiate between distinct value ranges for a reference property (in this case bending modulus of elasticity). The limits of mean modulus of elasticity ranged from $14000 \mathrm{~N} / \mathrm{mm}^{2}$ to $8600 \mathrm{~N} / \mathrm{mm}^{2}$ for the higher and lower visual grades, respectively (Sousa et al. 2014).

Bending properties were also considered as reference properties in the experimental campaign of Piazza and Riggio (2008), where hardness tests and ultrasound testing were used at the local scale for indirect measurement of bending stiffness. In that work, the results of both measurements as well as of visual grading have been compared to the stiffness as directly measured through bending tests. 


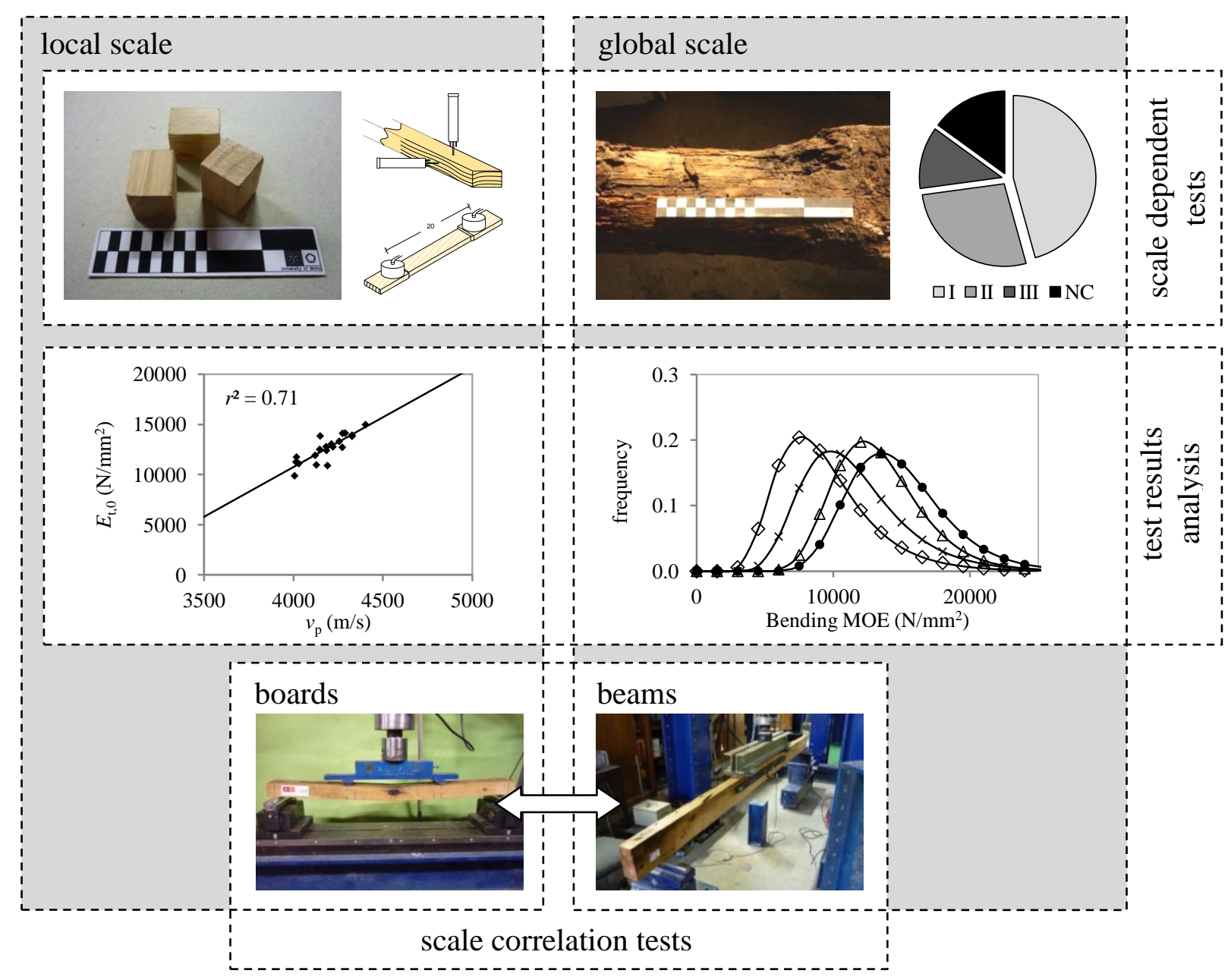

Figure 4: Sources and analysis of information regarding local and global scales.

In Figure 4, the type of tests considered in both scales were four-point bending tests as they allow to obtain direct information upon a reference property, namely the bending modulus of elasticity. In Sousa et al. (in-press), regarding a multi-scale experimental evaluation, strong correlations between local and global modulus of elasticity $\left(E_{\mathrm{m}, 1}\right.$ and $E_{\mathrm{m}, \mathrm{g}}$, respectively) were found within the same experimental phase $\left(r^{2}=0.82\right.$ in sawn beams and $r^{2}=0.89$ in sawn boards). Moderate to high correlations between $E_{\mathrm{m}, \mathrm{l}}$ and $E_{\mathrm{m}, \mathrm{g}}$ for different experimental phases $\left(r^{2}=0.68\right.$ and 0.71 , respectively) were found.

\subsection{Probability Assignment}

After gathering the initial data, it is necessary to hierarchically divide it in different levels of importance regarding the scope of the analysis. In parallel, it is necessary to convert the information into individual parameters that may efficiently represent it, without losing its significance. For that purpose, this methodology proposes to probabilistically define those properties with interest to the analysis, such as the expected mean value, variation and 
probability distribution. The layout of probabilistic models is often based on empirical data mostly combined with physical considerations, experience and good judgment. A classic procedure to assess if a given random variable may be well defined by a certain probability distribution, is the use of probability papers. Having selected a probability distribution family, the probability paper is an extremely useful tool for the purpose of checking the plausibility of the selected family (Faber, 2012). From probability papers, it is possible to assess the parameters of the inherent distribution with respect to the configuration of a given straight line (location and slope). However, a more efficient and accurate method is the Maximum Likelihood Method (Deublein et al., 2011), based on finding the set of parameters of an assumed probability distribution function which most likely characterizes the underlying data sample (Köhler, 2007). Maximum Likelihood estimates have been used by several authors to determine the probabilistic data parameters of timber's properties (Ditlevsen and Källsner, 1998; Fink and Köhler, 2011; Deublein et al. 2011), as well as its inherent uncertainty (Sousa et al. 2013b) and its tail distribution behaviour (Faber et al., 2004). The validity of the obtained parameters may afterwards be verified by use of $\chi^{2}$ goodness-of-fit tests.

This framework has also been based on the probabilistic model code (PMC) for timber structures issued by the Joint Committee for Structural Safety (JCSS), where stochastic resistance models for timber as a construction material are specified (JCSS, 2006). The stochastic models that characterize the mechanical properties of timber are described in that document, where from the knowledge of some specific properties, considered explicitly, one may obtain the others implicitly. Therefore, a full and precise application of this code requires the capability to predict these properties. The explicitly considered properties are defined as reference properties or also so-called key properties. These properties are generally chosen in accordance with visual stress-grading (Machado et al., 2011) or from tests carried with similar material (Toratti et al., 2007). In the case of the PMC, these properties, assumed as reference, are the bending strength $f_{m}$, bending modulus of elasticity $E_{m}$, and density $\rho_{m}$. The other mechanical properties of timber can be defined based in the reference properties through empirical expressions.

The models and values present in the PMC are intended as generic models in case no further detailed information is available. If further information is obtainable, the information in the code may be considered as the prior information in a Bayesian updating process.

Having determined the probabilistic parameters of the reference properties, its correlations to other properties and variation within scales, it is possible to predict the global properties of 
the element based on local information. To that aim, the material properties obtained through local testing are attributed to the clear wood sections, whereas the mechanical properties of sections with defects are reduced according to the visual grading results. After defining each section with its estimated properties, a global analysis, at the element scale, may be performed through linear elastic analysis. Given that the uncertainty and probabilistic variation of the properties are defined, it is also possible to locate sections and attribute the mechanical properties to the material in a random based method (Sousa et al. 2014).

By combination of the initial data with the prediction of the global properties it is possible to infer about different scenarios of prior data. In this framework, the use of Bayesian Probabilistic Networks (BPN) are considered as they establish a consistent framework between the prior information that is provided and the estimated results. Through the analysis of the conditional probabilities within the network, it is also possible to determine the causal relation (cause-effect) and dependencies between variables, therefore taking into account the expertise of the engineer. The common representation of a BPN consists in a directed acyclic graph, composed by a set of nodes, representing each system variable, connected by a set of directed edges, linking the variables as regards to their dependency or cause-effect relationship. Each variable node represents a random variable, which is either defined as a continuous random variable or as a finite set of mutually exclusive discrete states (intervals). The applicability and framework for construction of BPN in the field of reliability analysis has been addressed in e.g. Langseth and Portinale (2007) and Marquez et al. (2010), while in Deublein et al. (2011) hierarchical modelling through this method has been used to analyse the influence of different origins or dimensions of sawn structural timber on the relevant timber material properties accounting machine stress-grading indicators, and in Sousa et al. (2013a) for visual grading efficiency assessment.

At this point, the inference process should not be considered as an updating procedure, as it only considers the prior data that was used to perform the first prediction and classification of the global properties.

Figure 5 exemplifies this phase by considering the bending modulus of elasticity as the analyzed reference property. The probabilistic distribution is determined using probability papers that were also confirmed by maximum likelihood estimates. In this example, Lognormal distributions were considered due to its better fit to the experimental data (Sousa, 2013) and also attending to the recommendations given by the JCSS (2006). Then, the respective expected value and variation were determined with the cumulative distribution 
function of $E_{\mathrm{m}, \mathrm{g}}$ being similar in the different test scales (Sousa et al., in-press). The mean values of sawn boards bending tests were $18 \%$ and $7 \%$ higher than the equivalent values obtained for sawn beams, respectively for $E_{\mathrm{m}, 1}$ and $E_{\mathrm{m}, \mathrm{g}}$. In this example, the correlation between scales was analyzed considering the results of global and local modulus of elasticity in different scales as considered in Figures 3 and 4.

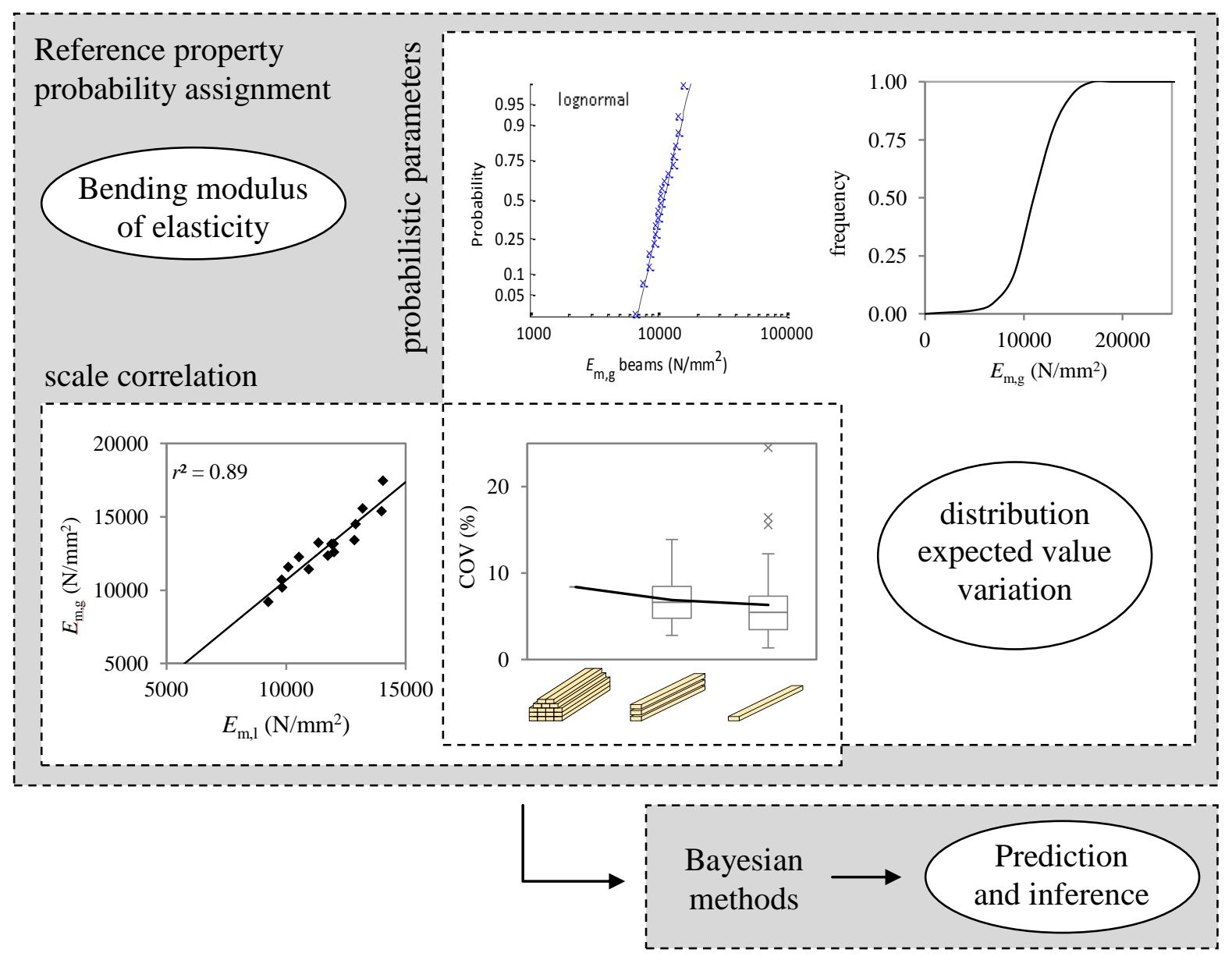

Figure 5: Example of probability assignment for a reference property and parameters for prediction and inference analysis.

Probabilistic based models were also considered in Tannert et al. (2010), where experimental investigations were carried out aiming on the development of a probabilistic model to predict the strength of dovetails connections considering the size effect. Also, in Tannert et al. (2011) a probabilistic model that accounted to the scale sensitivity of the material strength was proposed, which was modelled using Weibull statistics. 
In this example, correlation between scales is also considered by the analysis of the coefficient of variation (CoV) of the parameter in study. In Figure 5, that analysis represents the variation of this parameter in different scales for the measurement of ultrasound tests. The results of the ultrasound tests, that were correlated with bending stiffness in the previous phases of this proposed method, evidence a decrease in the mean value of $\mathrm{CoV}$ for lower size scales.

Besides the study of $\mathrm{CoV}$ in different scales, analysis of the variability of a given property may also be included by use of ANOVA tests, aiming at determining the importance of different parameters. This procedure was considered in Tannert and Haukaas (2013) for the analysis of the configuration of dovetail connections. Two levels of parameters were considered regarding 80 tests of single and double dovetail joints.

After defining the probabilistic parameter of the property in study, the final part of this phase considers the implementation of those results in an inference model by applying Bayesian methods.

\subsection{Long term structural analysis}

Long term structural analysis requires not only the assessment of the design conditions but also the continuous updating of the structures' present conditions as to take more substantiated decisions. Moreover, it is needed to predict or estimate the future performance of a structure in order to propose adequate maintenance or intervention plans. Figure 6 exemplifies this phase considering that new information was obtained through visual inspection and correlated with bending strength for different visual grades. This information will complement and update the prior existing knowledge available for the structural analysis of the timber elements.

The initial information may consider the design plans or information about the material characterization, however it is common not to have this information especially when dealing with historical constructions. In that case, it is needed to conduct a desk survey aiming at retrieving all possible information, such as historical documentation that may allow to determine when and how the structure was made and what materials were used. However, when even this information is not available, an initial preliminary visual survey is necessary, which will be then complemented with the experience based opinion of the inspector about the condition of the timber elements. This initial information should consider the assessment of the structure regarding the construction methods that were employed when the building 
was made and also comparison to similar construction systems or buildings of the same period and/or region. This initial information must also consider the geometric survey of the elements as to obtain a first design plan of the structural model. After obtaining this preliminary data, a more careful inspection should be considered. In Figure 6, the material characterization was complemented with the information of visual inspection, allowing to differentiate the elements in visual grades with significant differences in the mean bending strength. By consideration of different hypothesis for the visual grading, the probabilistic safety assessment resulted in distinct reliability indexes (Sousa, 2013). For example, when the timber element is visually graded as class I, the reliability index is 5.82, while it may decrease down to 1.33 when the element is considered not suitable for structural use. This information, combined with the use of decay models (Lourenço et al. 2013), may be used in the prediction of the future performance of the element, and therefore be useful in the definition of maintenance and intervention planning.

The consideration of local data, obtained through experimental tests, for the determination of the global behaviour of the element was also considered in Branco et al. (2010) and in Riggio et al. (2014). On both that studies, the results were then used to infer on the possibility and performance of possible intervention and strengthening techniques. Meanwhile in Tannert and Haukaas (2013), the possibility of updating a model for the prediction of the structural performance of dovetail joints was developed with Bayesian techniques, as it is also considered in the example proposed in Figure 6.

Following, general description regarding the main steps of this phase are provided. 


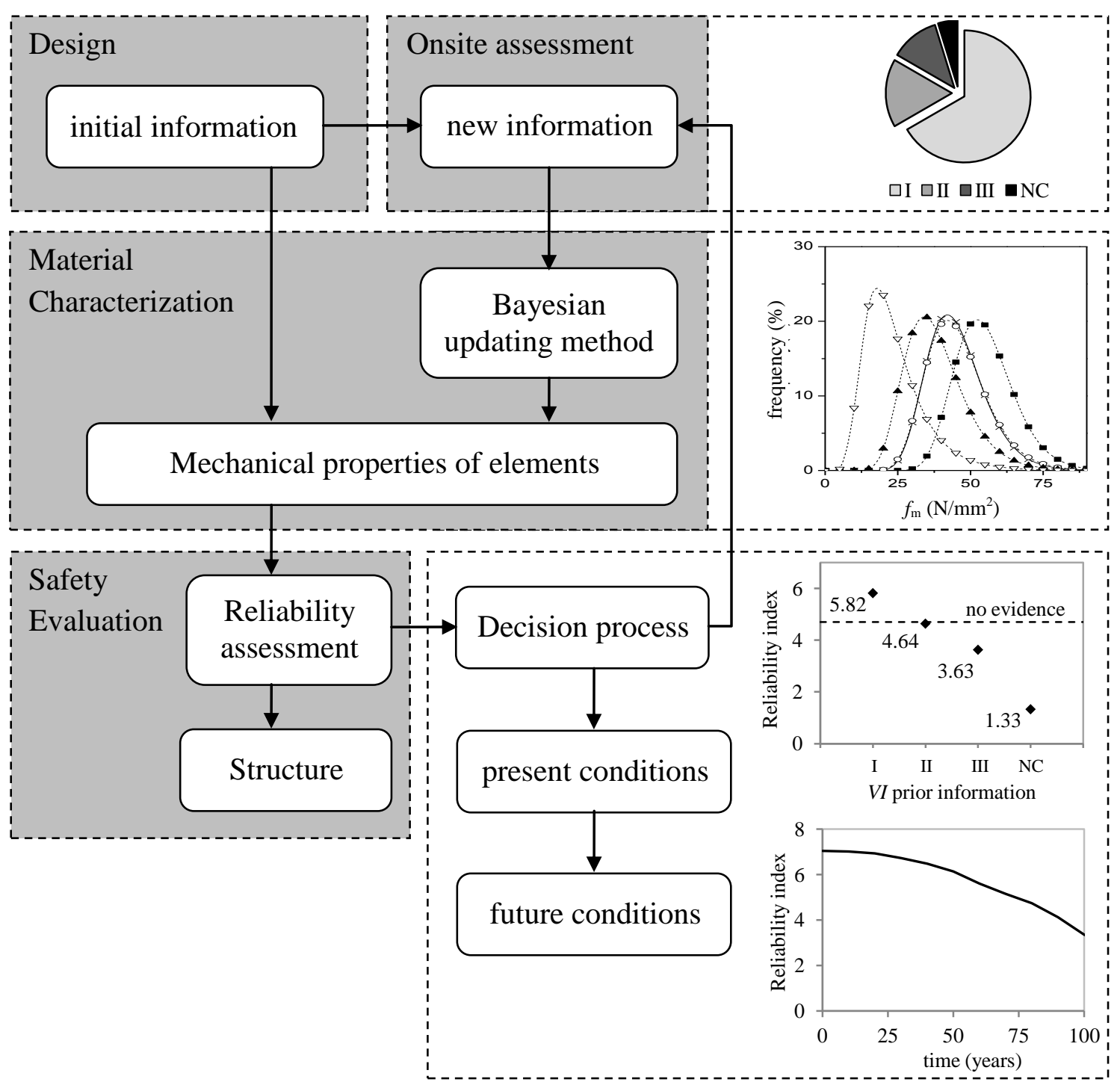

Figure 6: Schematic procedure for a decision process considering an updating process.

\subsubsection{Reliability assessment}

Stochastic reliability methods due to their probabilistic nature, when applied to structural engineering problems, allow to consider a large amount of information about the basic variables involved in the safety assessment of an existing structure. In structural reliability applications, it is often necessary to consider the characteristic values of demand and resistance, and thus in the large majority of cases the solution is found in the probability distribution extremes. This type of problems is usually denominated as a tail sensitivity problem. Accounting this premise, the probability of failure is extremely sensitive to the probabilistic parameters chosen for the probability distribution, and thus the importance of 
correctly defining and calibrating the probabilistic model according to the existing data in the previous steps of the analysis.

The detailing of the specific algorithms for different reliability assessment is outside the scope of this work, as they are firmly well established in literature for existing structures (Schneider, 1997; Faber, 2000). Nevertheless, specific concerns for the assessment of the mechanical properties of timber structures should be taken in consideration as described in (Larsen, 2001; Köhler et al. 2007; Lourenço et al., 2013; Rosowsky, 2013), as well as for the load effects (Sørensen et al. 2005).

\subsubsection{Decision process}

Based on the initial reliability analysis, the assessment engineer may take different decisions regarding the need or not to intervene on the existing timber structure and, in case it is needed, what kind of intervention is more adequate. The proposed methodology also allows to define different maintenance plans, as in the inference level it is possible to assess different scenarios and even to implement a time dependent analysis provided that dynamic probabilistic networks are assumed.

Since this methodology allows for the implementation of the engineers' experience along the different steps of the analysis, the results of the assessment may slightly differ attending to different levels of expertise. To minimize that effect, an updating process is considered where through the consideration of new information the updated prediction and inference can be more precise and adequate to the structure in analysis. The incorporation of an updating process allows that information retrieved from a monitoring system can also be implemented in the assessment procedure without need to initiate the analysis from the starting point.

\subsubsection{Updating}

Throughout their lifetime structures change due to many aspects, from natural causes (such as material deterioration, environment exposure and long term effects of loads in structures), to human decisions (such as modification of the structure or change of use) or even by accidental actions, only to point a few. Thus, the assessment of existing structures should be regarded as a successive process of model updating and consequent evaluation regarding new information. This information may also be retrieved from monitoring systems to which an assessment methodology that supports the possibility of continuous updating may be required. 
In this methodology, Bayesian methods are considered for parameter and model updating as they allow quantifying an approximation about the statistical uncertainty related to the estimated parameters, regarding both the physical uncertainty of the considered variable, as well as the statistical uncertainty related to the model parameters. However, for making this possible, it is necessary to take into account the measurement and the model uncertainties in the probabilistic model formulation. The fundaments for updating have been described in (Faber, 2000) and applied for updating timber elements' reference properties in Machado et al. (2011) and also considering NDT data in Sousa et al. (2013b) for assessment of different decay scenarios, and in Fink and Köhler (2014b) for updating the load and resistance properties of a partly failed timber construction based on different kind of information.

In this methodology, the updating scheme is based on different Bayesian methods which results may produce changes at different steps of the assessment. When new information is known for the local mechanical properties of the timber element by consequence of new tests or data sources, therefore independent data from the prior analysis, the updating process leads to the second step of the proposed methodology. This process is made through Bayes' theorem, updating the prior distribution functions into posterior distribution functions. Otherwise, when new information leads only to the change in the global analysis of the element, the updating may be made through inference on the Bayesian Probabilistic Network at the inference level.

\section{DISCUSSION}

The previously described methodology for assessment of timber elements is based in four main steps: (i) scale of information; (ii) local and global data; (iii) probability assignment; and (iv) long term structural analysis. All steps require different levels of knowledge upon wood science and timber engineering. The first two steps are specifically related to the correlation between scales, selection of tests and source of information which is a common starting point to other evaluation methodology. The innovation of this methodology, compared to others, resides in the possibility of combining this information in a probabilistic framework allowing for the prediction, inference and updating of the reference properties of timber elements, within a holistic framework.

The prior information considered in this methodology was chosen accordingly to usual available data and common practice, as it intends to be used not only for heritage structures, where more resources can be applied, but also in regular existing timber structures. 
Nevertheless, even if the proposed methodology suggests to combine information at the material scale by sampling of small size clear wood specimens combined with NDT and visual inspection at the element scale, it also allows to combine information from different sources, such as from load-proof tests or destructive testing, just by adding another scale in the first step of the analysis and its correlations to the other scales.

Numerical modeling information, even if not resulting from a probabilistic approach, may also be considered for the prediction and inference of the global properties or otherwise considered as posterior information in the updating process, by consideration of different levels of importance for the prior information.

A requirement to the implementation of this methodology, as in other probabilistic based assessments, is that a large amount of data is needed. In this case a significant effort is required for the construction of a database for the determination of the reference properties. Nevertheless, by consideration of the correlations between scales it is possible to calibrate the present methodology for the case where only scarce information on one scale is available, provided that a prior database is available for similar conditions.

One advantage of the methodology is that the level of information may be adapted according to the importance or need of the analysis, and after it may be updated with newer or more complete information.

The proposed methodology adopts a combination of several techniques aiming at the assessment of existing timber elements accounting information from different sources, and it is suggested to be integrally followed as a complete assessment tool. However, attending to the specificities of the analysis, one may choose to favor one specific procedure or step within the methodology. This is also taken into consideration in this methodology, as it is possible to attribute different levels of belief and importance to each one of the steps taking into account its Bayesian nature.

\section{CONCLUSIONS}

The mechanical properties of timber elements may be assessed individually by different techniques, however the analysis of the global mechanical performance of a timber element requires a holistic methodology capable of combining the information from different sources, as well as to attend to different load scenarios or level of conservation. In this work, a probabilistic based methodology was proposed taking into account the combination of information retrieved in both material and structural scale. To that aim, the presented 
methodology evidenced the possibility of using sampling of clear wood specimens for representation of the material scale combined with results of visual grading at the structural scale. Also, the variation within the same scale and correlation between different scales were taken into account in the proposed methodology.

Firstly, the initial data is probabilistically parameterized and the reference properties are selected attending to the scope of the analysis. Following, the methodology proposes to predict and infer about the global properties based on the variation and distribution of the local mechanical properties and visual grading of the elements.

The methodology's final step considers the assessment of the timber element through a reliability analysis described along this work, which poses as the defining parameter for the decision process of the assessment engineer. Ultimately, the decision process may be aided by new information implemented through a Bayesian updating scheme that allows to act and influence at different levels of the assessment methodology.

Although the proposed methodology is based on reliability and probabilistic analysis, it is also compatible with information obtained deterministically through other sources than those presented here, as well to be modified as to privilege different types and objectives of assessment, thus posing as a viable solution not only for heritage timber structures but also to other existing timber structural structures.

\section{ACKNOWLEDGMENTS}

The financial support of the Portuguese Science Foundation (Fundação de Ciência e Tecnologia, FCT), through PhD Grant SFRH/BD/62326/2009, is gratefully acknowledged.

\section{REFERENCES}

Alessandri, C., Mallardo, V., Pizzo, B., and Ruocco, E. 2012. The roof of the Church of the Nativity in Bethlehem: Structural problems and intervention techniques. J. Cult. Herit., 13(4):e70-e81.

ASTM D143. 2000. Standard test methods for small clear specimens of timber, American Society for Testing Materials, EUA.

Branco, J. M., Piazza, M., and Cruz, P. J. 2010. Structural analysis of two King-post timber trusses: Non-destructive evaluation and load-carrying tests. Constr. Build. Mater., 24(3):371-383. 
Calderoni, C., De Matteis, G., Giubileo, C., and Mazzolani, F. M. 2010. Experimental correlations between destructive and non-destructive tests on ancient timber elements. Eng. Struct., 32(2):442-448.

Calderoni, C., De Matteis, G., Giubileo, C., and Mazzolani, F. M. 2006. Flexural and shear behaviour of ancient wooden beams: Experimental and theoretical evaluation. Eng. Struct., 28(5):729-744.

Cavalli, A., and Togni, M. 2013. How to improve the on-site MOE assessment of old timber beams combining NDT and visual strength grading. Nondestruct. Test. Eva., 28(3):252262.

CEN 2004. EN 1995-1-1:2004, Eurocode 5: design of timber structures. Part 1-1: General common rules and rules for buildings, European Committee for Standardization, Brussels.

Cointe, A., Castéra, P., Morlier, P., and Galimard, P. 2007. Diagnosis and monitoring of timber buildings of cultural heritage. Struct. Saf., 29(4):337-348.

Cruz, H., Yeomans, D., Tsakanika, E., Macchioni, N., Jorissen, A., Touza, M., Mannucci, M., and Lourenço, P. B. 2013. Guidelines for the on-site assessment of historic timber structures. Int. J. Archit. Herit., DOI: 10.1080/15583058.2013.774070.

Dackermann ,U., Crews, K., Kasal, B., Li, J., Riggio, M., Rinn, F., and Tannert, T. 2014. In situ assessment of structural timber using stress-wave measurements. Mater. Struct., 47(5):787-803.Deublein, M., Schlosser, M., and Faber, M. H. 2011. Hierarchical modelling of structural timber material properties by means of Bayesian Probabilistic Networks. Applications of Statistics and Probability in Civil Engineering. Edited by: Faber, Köhler and Nishijima. Taylor \& Francis Group, London. 1377-1385.

Ditlevsen, O. D., and Källsner, B. 1998. System effects influencing the bending strength of timber beams. In Reliability and Optimization of Structural Systems, Proceedings of 8th IFIP WG 7.5 Working Conference, 129-136. Krakow, Poland

Faber, M. H. 2000. Reliability based assessment of existing structures. Prog. Struct. Eng. Mat., 2(2):247-253.

Faber, M. H. 2012. Statistics and Probability Theory: In Pursuit of Engineering Decision Support. Springer. ISBN: 9400740557.

Faber, M. H., Köhler, J., and Sørensen, J. D. 2004. Probabilistic modelling of graded timber material properties. Struct. Saf., 26(3):295-309. 
Faggiano, B., Grippa, M. R., Marzo, A., and Mazzolani, F. M. 2011. Experimental study for non-destructive mechanical evaluation of ancient chestnut timber. J. Civ. Struct. Health Monitor., 1(3-4):103-112.

Feio, A. O., Lourenço, P. B., and Machado, J. S. 2007. Non-destructive evaluation of the mechanical behaviour of chestnut wood in tension and compression parallel to grain. Int. J. Archit. Herit.,1(3):272-292.

Fink, G., and Köhler, J. 2011. Multiscale variability of stiffness properties of timber boards. ICASP Applications of Statistics and Probability in Civil Engineering. Edited by Faber, Köhler and Nishijima. Taylor \& Francis Group, London. 1369-1376.

Fink, G., and Köhler, J. 2014a. Model for the prediction of the tensile strength and tensile stiffness of knot clusters within structural timber. Eur. J. Wood Wood Prod., 72(3):331341.

Fink, G., and Köhler, J. 2014b. Risk Based Investigations of Partly Failed or Damaged Timber Constructions. Materials and Joints in Timber Structures, RILEM Bookseries 9. Springer Netherlands.

Isaksson, T. 1999. Modelling the variability of bending strength in structural timber. Report TVBK-1015, Dept. of Structural Engineering, Lund University, Sweden.

ISO 13910. 2005. Structural timber - Characteristic values of strength-graded timber Sampling, full-size testing and evaluation. International Organization for Standardization, Geneva.

ISO 3129. 2012. Wood - Sampling methods and general requirements for physical and mechanical tests. International Organization for Standardization, Geneva.

JCSS 2006. JCSS Probabilistic Model Code, Part 3: Resistance Models - 3.5 Properties of Timber. Probabilistic Model Code, Joint Committee on Structural Safety, Internet Publication: www.jcss.ethz.ch.

Kasal, B. 2010. In Situ Assessment of Structural Timber: State-of-the-Art, Challenges and Future Directions. Adv. Mater. Res., 133:43-52.

Kasal, B., and Anthony, R. W. 2004. Advances in in situ evaluation of timber structures. Prog. Struct. Eng. Mat., 6(2):94-103.

Kasal, B., and Tannert, T. 2010. In situ assessment of structural timber. RILEM state of the art reports.

Köhler, J. 2007. Reliability of timber structures. $\mathrm{PhD}$ thesis, Institute of Structural Engineering Swiss Federal Institute of Technology, Zurich, Switzerland. 
Köhler, J., Sørensen, J. D., and Faber, M. H. 2007. Probabilistic modeling of timber structures. Struct. Saf., 29(4):255-267.

Langseth, H., and Portinale, L. 2007. Bayesian networks in reliability. Reliab. Eng. Syst. Saf., 92(1):92-108.

Larsen, H. J. 2001. Properties affecting reliability design of timber structures. In Cost E24 Seminar on Reliability of timber structures, 1-26. Coimbra, Portugal.

Lourenço, P. B., Feio, A. O., and Machado, J. S. 2007. Chestnut wood in compression perpendicular to the grain: non-destructive correlations for test results in new and old wood. Constr. Build. Mater., 21(8):1617-1627.

Lourenço, P. B., Sousa, H. S., Brites, R. D., and Neves, L. C. 2013. In situ measured cross section geometry of old timber structures and its influence on structural safety. Mater. Struct., 46(7):1193-1208.

Macchioni, N., Brunetti, M., Pizzo, B., Burato, P., Nocetti, M., and Palanti, S. 2012. The timber structures in the Church of the Nativity in Bethlehem: Typologies and diagnosis. J. Cult. Herit., 13(4):e42-e53.

Machado, J. S. 2013. In Situ Evaluation of the Reference Properties of Structural Timber Members. Use of Available Tools and Information. Adv. Mat. Res., 778:137-144.

Machado, J. S., and Palma, P. 2011. Non-destructive evaluation of the bending behaviour of in-service pine timber structural elements. Mater. Struct., 44(5):901-910.

Machado, J. S., Lourenço, P. B., and Palma, P. 2011. Assessment of the structural properties of timber members in situ: a probabilistic approach. In Proceedings of SHATIS 11. Lisbon, Portugal.

Marquez, D., Neil, M., and Fenton, N. 2010. Improved reliability modelling using Bayesian networks and dynamic discretization. Reliab. Eng. Syst. Saf., 95(4):412-425.

Piazza, M., and Riggio, M. 2008. Visual strength-grading and NDT of timber in traditional structures. J. Build. Appraisal, 3(4):267-296.

Riberholt, H., and Madsen, P. H. 1979. Strength of timber structures, measured variation of the cross sectional strength of structural lumber. Report R 114, Struct. Research Lab., Technical University of Denmark.

Riggio, M., Anthony, R. W, Augelli, F., Kasal, B., Lechner, T., Muller, W., and Tannert, T. 2014a. In situ assessment of structural timber using nondestructive techniques. Mater. Struct., 47(5):749-766. 
Riggio, M., Tomasi, R., and Piazza, M. 2014b. Refurbishment of a Traditional Timber Floor with a Reversible Technique: Importance of the Investigation Campaign for Design and Control of the Intervention. Int. J. Archit. Herit., 8(1):74-93.

Rosowsky, D. V. 2013. Evolution of probabilistic analysis of timber structures from secondmoment reliability methods to fragility analysis. Struct. Saf., 41:57-63.

Ross, R., Brashaw, B., and Pellerin, R. 1998. Non-destructive evaluation of wood. Forest Prod. J., 48(1):101-105.

Schneider, J. 1997. Introduction to safety and reliability of structures. Structural Engineering documents 5. IABSE, Zurich, Switzerland.

Sørensen, J. D., Svensson, S., and Stang, B. D. 2005. Reliability-based calibration of load duration factors for timber structures. Struct. Saf., 27(2):153-169.

Sousa, H. S, Branco, J. M and Lourenço, P. B. (in-press). Use of bending tests and visual inspection for multi-scale experimental evaluation of chestnut timber beams stiffness. J. Civ. Eng. Manag., doi:10.3846/13923730.2014.914083.

Sousa, H. S. 2013. Methodology for safety evaluation of existing timber elements. PhD thesis, Civil Engineering Department, University of Minho, Guimarães, Portugal

Sousa, H. S., Branco, J. M., and Lourenço, P. B. 2013a. Effectiveness and subjectivity of visual inspection as a method to assess bending stiffness and strength of chestnut elements. Adv. Mater. Res. 778:175-182.

Sousa, H. S., Branco, J. M., and Lourenço, P. B. 2014. Prediction of global bending stiffness of timber beams by local sampling data and visual inspection. European Eur. J. Wood Wood Prod., 72:453-461.

Sousa, H. S., Sørensen, J. D., Kirkegaard, P. H., Branco, J. M., and Lourenço, P. B. 2013 b. On the use of NDT data for reliability-based assessment of existing timber structures. Eng. Struct., 56:298-311.

Tampone, G., Mannucci, M., Macchioni, N., and Gambetta, A. 2002. Strutture di legno: cultura, conservazione, restauro. De Lettera. Milan, Italy.

Tannert, T., and Haukaas, T. 2012. Probabilistic models for structural performance of rounded dovetail joints. J. Struct. Eng., 139(9):1478-1488.

Tannert, T., Anthony, R. W., Kasal, B., Kloiber, M., Piazza, M., Riggio, M., Rinn, F., Widmann, R., and Yamaguchi, N. 2014. In situ assessment of structural timber using semi-destructive techniques. Mater. Struct., 47(5):767-785. 
Tannert, T., Lam, F., and Vallée, T. (2011). Structural performance of rounded dovetail connections: experimental and numerical investigations. European Eur. J. Wood Wood Prod., 69(3):471-482.

Tannert, T., Lam, F., and Vallée, T. 2010. Strength prediction for rounded dovetail connections considering size effects. J. Eng. Mech., 136(3):358-366.

Toratti, T., Schnabl, S., and Turk, G. 2007. Reliability analysis of a glulam beam. Struct. Saf., 29(4):279-293.

UNI 11119. 2004. Cultural heritage - Wooden artifacts - Load-bearing structures - On site inspections for the diagnosis of timber members. Milan, Italy: ENIU.

Zona, A., Barbato, M., and Fragiacomo, M. 2011. Finite-element model updating and probabilistic analysis of timber-concrete composite beams. J. Struct. Eng., 138(7):899910. 